\title{
The Carathéodory Inequality on the Boundary for Holomorphic Functions in the Unit Disc
}

\author{
B.N. Örnek \\ Department of Computer Engineering, Amasya University \\ Merkez-Amasya 05100, Turkey \\ E-mail: nafiornek@gmail.com
}

Received February 12, 2013, revised December 13, 2015

\begin{abstract}
In this paper, a boundary version of the Carathéodory inequality is studied. For the function $f(z)$, defined in the unit disc with $f(0)=0$, $\Re f(z) \leq A$, we estimate a modulus of angular derivative at the boundary point $z_{0}, \Re f\left(z_{0}\right)=A$, by taking into account the first two nonzero Maclaurin coefficients. The sharpness of these estimates is also proved.

Key words: Schwarz lemma at the boundary, Carathéodory inequality.

Mathematics Subject Classification 2010: 30C80.
\end{abstract}

\section{Introduction}

In recent years, a boundary version of Schwarz lemma was studied by D. Burns and S.G. Krantz ([2]), R.Osserman ([7]), V. N. Dubinin ([4]), B. N. Örnek ([8]) and others. On the contrary there was published the book ([1]), where the authors studied the sharp real-part theorems (in particular, the Carathéodory inequalities), which are frequently used in the theory of entire functions and in the analytic function theory.

The Carathéodory inequality states that if the function $f$ is holomorphic in the unit disc $D=\{z:|z|<1\}$ with $f(0)=0$ and $\Re f \leq A$ in $D$, then the inequality

$$
|f(z)| \leq \frac{2 A r}{1-r}, \quad|z|=r
$$

holds for all $z \in D$, and moreover,

$$
\left|f^{\prime}(0)\right| \leq 2 A
$$

(C) B.N. Örnek, 2016 
The equality is achieved in (1.1) (for some nonzero $z \in D$ ) or in (1.2) if and only if $f$ is a function of the form

$$
f(z)=\frac{2 A z e^{i \theta}}{1+z e^{i \theta}}
$$

where $\theta$ is a real number ([1], p. 3,4$)$.

On the other hand, if $f(z)=c_{p} z^{p}+c_{p+1} z^{p+1} \ldots p \in \mathbb{N}$ is a holomorphic function in the unit disc $D=\{z:|z|<1\}$ and if $\Re f \leqslant A$ for $|z|<1$, it can be seen that the Carathéodory inequality can be strengthened by standard methods:

$$
|f(z)| \leq \frac{2 A|z|^{p}}{1-|z|^{p}}
$$

and

$$
\left|c_{p}\right| \leq 2 A .
$$

The classical Schwarz lemma states that a holomorphic function $f$, mapping the unit disc $D$ into itself with $f(0)=0$, satisfies the inequality $|f(z)| \leq|z|$ for any point $z \in D$, and $\left|f^{\prime}(0)\right| \leq 1$. The equality in these inequalities (in the first one, for $z \neq 0$ ) occurs only if $f(z)=z e^{i \theta}, \theta$ is a real number (see [5]). From the Schwarz lemma, it is known that if a holomorphic function $f$, mapping the unit disc into itself with $f(0)=0$, extends continuously to a boundary point $z_{0}$ with $\left|z_{0}\right|=1,\left|f\left(z_{0}\right)\right|=1$, and $f^{\prime}\left(z_{0}\right)$ exists, then $\left|f^{\prime}\left(z_{0}\right)\right| \geq 1$. This result of the Schwarz lemma and its generalization are described in literature as the Schwarz lemma at the boundary.

In ([7]), R. Osserman offered the following boundary refinement of the classical Schwarz lemma. It is very much in the spirit of the sort of result we wish to consider here. That is,

$$
\left|f^{\prime}\left(z_{0}\right)\right| \geqslant \frac{2}{1+\left|f^{\prime}(0)\right|}
$$

and

$$
\left|f^{\prime}\left(z_{0}\right)\right| \geq 1
$$

under the assumption $f(0)=0$, where $f$ is a holomorphic function mapping the unit disc into itself and $z_{0}$ is a boundary point to which $f$ extends continuously, and $\left|f\left(z_{0}\right)\right|=1$. Moreover, the equality in (1.4) holds if and only if $f(z)=z e^{i \theta}$, where $\theta$ is a real number. Also, $z_{0}=1$ in inequality (1.3), the equality occurs for the function $f(z)=z(z+a) /(1+a z), 0 \leq a \leq 1$.

If, in addition, the function $f$ has an angular limit $f\left(z_{0}\right)$ at $z_{0} \in \partial D,\left|f\left(z_{0}\right)\right|=$ 1 , then by Julia-Wolff lemma, the angular derivative $f^{\prime}\left(z_{0}\right)$ exists, and $1 \leq$ $\left|f^{\prime}\left(z_{0}\right)\right| \leq \infty([9])$. 
Moreover, if $f(z)=c_{p} z^{p}+c_{p+1} z^{p+1} \ldots .$, then

$$
\left|f^{\prime}\left(z_{0}\right)\right| \geqslant p+\frac{1-\left|c_{p}\right|}{1+\left|c_{p}\right|}
$$

Moreover, the equality in (1.5) occurs for the function $f(z)=z^{p}(z+\gamma) /(1+\gamma z)$, $0 \leq \gamma \leq 1$

We studied the boundary Carathéodory inequalities as an analog of the boundary Schwarz lemma. We estimated a modulus of angular derivative of the function that satisfied the Carathéodory inequality by taking into account the first two nonzero Maclaurin coefficients.

\section{Main Results}

We have the following results.

Theorem 1. Let $f(z)=c_{p} z^{p}+c_{p+1} z^{p+1} \ldots, c_{p} \neq 0, p \geq 2, p \in \mathbb{N}$, be a holomorphic function in the unit disc $D$ and let $\Re f \leqslant A$ for $|z|<1$. Further, assume that for some $z_{0} \in \partial D, f$ has an angular limit $f\left(z_{0}\right)$ at $z_{0}, \Re f\left(z_{0}\right)=A$. Then the angular derivative $f^{\prime}\left(z_{0}\right)$ exists, and

$$
\left|f^{\prime}\left(z_{0}\right)\right| \geqslant \frac{A}{2}\left(p+\frac{2\left(2 A-\left|c_{p}\right|\right)^{2}}{4 A^{2}-\left|c_{p}\right|^{2}+2 A\left|c_{p+1}\right|}\right) .
$$

Moreover, the equality in (1.6) occurs for the function

$$
f(z)=2 A \frac{z^{p}}{1+z^{p}} .
$$

P r o o f. Consider the functions

$$
w(z)=\frac{f(z)}{2 A-f(z)}, \quad B(z)=z^{p}
$$

The functions $w(z)$ and $B(z)$ are holomorphic in $D$, and $|w(z)| \leq 1,|B(z)|<1$ for $|z|<1$.

That is,

$$
\begin{aligned}
|2 A-f(z)|^{2} & =|f(z)-2 A|^{2}=|f(z)|^{2}-2 \Re(f(z) 2 A)+4 A^{2} \\
& =|f(z)|^{2}-4 A \Re(f(z))+4 A^{2} .
\end{aligned}
$$

From the hypothesis, since $\Re f(z) \leqslant A$ and $4 A \Re f(z) \leqslant 4 A^{2}$, we consider

$$
|2 A-f(z)|^{2} \geq|f(z)|^{2}-4 A \Re f(z)+4 A \Re f(z)=|f(z)|^{2} .
$$


Therefore, we obtain

$$
\left|\frac{f(z)}{2 A-f(z)}\right| \leq 1
$$

By the maximum principle, for each $z \in D$, we have

$$
|w(z)| \leq|B(z)|
$$

Therefore,

$$
t(z)=\frac{w(z)}{B(z)}
$$

is a holomorphic function in $D$, and $|t(z)| \leq 1$ for $|z| \leq 1$. In particular, we have

$$
|t(0)|=\frac{\left|c_{p}\right|}{2 A} \leq 1
$$

and

$$
\left|t^{\prime}(0)\right|=\frac{\left|c_{p+1}\right|}{2 A}
$$

If $|t(0)|=1$, then by the maximum principle, we have $\frac{w(z)}{B(z)}=e^{i \theta}$ and $f(z)=$ $\frac{2 A z^{p} e^{i \theta}}{1+z^{p} e^{i \theta}}$, where $\theta$ is a real number. For the function $f(z),(1.6)$ holds. Further we may assume $f(z) \not \equiv \frac{2 A z^{p} e^{i \theta}}{1+z^{p} e^{i \theta}}$, and thus $|t(0)|<1$.

Moreover, since the expression $\frac{z_{0} w^{\prime}\left(z_{0}\right)}{w\left(z_{0}\right)}$ is a real number greater than or equal to 1 (see [7]) and $\Re f\left(z_{0}\right)=A$ yields $\left|w\left(z_{0}\right)\right|=1$, we get

$$
\frac{z_{0} w^{\prime}\left(z_{0}\right)}{w\left(z_{0}\right)}=\left|\frac{z_{0} w^{\prime}\left(z_{0}\right)}{w\left(z_{0}\right)}\right|=\left|w^{\prime}\left(z_{0}\right)\right| .
$$

Also, since $|w(z)| \leq|B(z)|$, we take

$$
\frac{1-|w(z)|}{1-|z|} \geq \frac{1-|B(z)|}{1-|z|}
$$

Because $f(z)$ has an angular limit at $z_{0}$, then $w(z)$ has an angular limit at $z_{0}$ and from the Julia-Wolff lemma the function $w(z)$ has an angular derivative at $z_{0}$. Passing to the angular limit in the last inequality yields

$$
\left|w^{\prime}\left(z_{0}\right)\right| \geq\left|B^{\prime}\left(z_{0}\right)\right|
$$

Therefore, we obtain

$$
\frac{z_{0} w^{\prime}\left(z_{0}\right)}{w\left(z_{0}\right)}=\left|w^{\prime}\left(z_{0}\right)\right| \geq\left|B^{\prime}\left(z_{0}\right)\right|=\frac{z_{0} B^{\prime}\left(z_{0}\right)}{B\left(z_{0}\right)} .
$$


The composite function

$$
T(z)=\frac{t(z)-t(0)}{1-\overline{t(0)} t(z)}
$$

is holomorphic in the unit disc $D,|T(z)|<1, T(0)=0$, and $\left|T\left(z_{0}\right)\right|=1$ for $z_{0} \in \partial D$.

From (1.3), we obtain

$$
\begin{aligned}
\frac{2}{1+\left|T^{\prime}(0)\right|} & \leq\left|T^{\prime}\left(z_{0}\right)\right|=\frac{1-|t(0)|^{2}}{\left|1-\overline{t(0)} t\left(z_{0}\right)\right|^{2}}\left|t^{\prime}\left(z_{0}\right)\right| \\
& =\frac{1-|t(0)|^{2}}{\left|1-\overline{t(0)} t\left(z_{0}\right)\right|^{2}}\left|\frac{w^{\prime}\left(z_{0}\right)}{B\left(z_{0}\right)}-\frac{w\left(z_{0}\right) B^{\prime}\left(z_{0}\right)}{B^{2}\left(z_{0}\right)}\right| \\
& =\frac{1-|t(0)|^{2}}{\left|1-\overline{t(0)} t\left(z_{0}\right)\right|^{2}}\left|\frac{w\left(z_{0}\right)}{z_{0} B\left(z_{0}\right)}\right|\left|\frac{z_{0} w^{\prime}\left(z_{0}\right)}{w\left(z_{0}\right)}-\frac{z_{0} B^{\prime}\left(z_{0}\right)}{B\left(z_{0}\right)}\right| \\
& \leq \frac{1+|t(0)|}{1-|t(0)|}\left\{\left|w^{\prime}\left(z_{0}\right)\right|-\left|B^{\prime}\left(z_{0}\right)\right|\right\}
\end{aligned}
$$

and

$$
\frac{2}{1+\left|T^{\prime}(0)\right|} \leq \frac{1+|t(0)|}{1-|t(0)|}\left\{\left|w^{\prime}\left(z_{0}\right)\right|-\left|B^{\prime}\left(z_{0}\right)\right|\right\} .
$$

It can be seen that

$$
\begin{aligned}
T^{\prime}(z) & =\frac{1-|t(0)|^{2}}{(1-\overline{t(0)} t(z))^{2}} t^{\prime}(z) \\
T^{\prime}(0) & =\frac{1-|t(0)|^{2}}{\left(1-|t(0)|^{2}\right)^{2}} t^{\prime}(0) \\
& =\frac{t^{\prime}(0)}{1-|t(0)|^{2}}
\end{aligned}
$$

and

$$
\left|T^{\prime}(0)\right|=\frac{\left|t^{\prime}(0)\right|}{1-|t(0)|^{2}}=\frac{\frac{\left|c_{p+1}\right|}{2 A}}{1-\left(\frac{\left|c_{p}\right|}{2 A}\right)^{2}}=\frac{2 A\left|c_{p+1}\right|}{4 A^{2}-\left|c_{p}\right|^{2}} .
$$

Since $w(z)$ has an angular derivative at $z_{0}$, the function $f(z)$ has an angular derivative at $z_{0}$. Thus, we take

$$
\left|w^{\prime}\left(z_{0}\right)\right|=\frac{2 A\left|f^{\prime}\left(z_{0}\right)\right|}{\left|2 A-f\left(z_{0}\right)\right|^{2}} .
$$


Also, we have $\left|B^{\prime}\left(z_{0}\right)\right|=p$ for $z_{0} \in \partial D$. Let us substitute the values of $\left|T^{\prime}(0)\right|$, $\left|w^{\prime}\left(z_{0}\right)\right|,\left|B^{\prime}\left(z_{0}\right)\right|$ and $|t(0)|$ into (1.8). Therefore, we obtain

$$
\begin{aligned}
& \frac{2}{1+\frac{2 A\left|c_{p+1}\right|}{4 A^{2}-\left|c_{p}\right|^{2}}} \leq \frac{1+\frac{\left|c_{p}\right|}{2 A}}{1-\frac{\left|c_{p}\right|}{2 A}}\left\{\frac{2 A\left|f^{\prime}\left(z_{0}\right)\right|}{\left|2 A-f\left(z_{0}\right)\right|^{2}}-p\right\} \\
&=\frac{2 A+\left|c_{p}\right|}{2 A-\left|c_{p}\right|}\left\{\frac{2 A\left|f^{\prime}\left(z_{0}\right)\right|}{\left|2 A-f\left(z_{0}\right)\right|^{2}}-p\right\}, \\
& \frac{2\left(4 A^{2}-\left|c_{p}\right|^{2}\right)}{4 A^{2}-\left|c_{p}\right|^{2}+2 A\left|c_{p+1}\right|} \frac{2 A-\left|c_{p}\right|}{2 A+\left|c_{p}\right|} \leq \frac{2 A\left|f^{\prime}\left(z_{0}\right)\right|}{\left|2 A-f\left(z_{0}\right)\right|^{2}}-p
\end{aligned}
$$

and

$$
\frac{2\left(4 A^{2}-\left|c_{p}\right|^{2}\right)}{4 A^{2}-\left|c_{p}\right|^{2}+2 A\left|c_{p+1}\right|} \frac{2 A-\left|c_{p}\right|}{2 A+\left|c_{p}\right|}+p \leq \frac{2 A\left|f^{\prime}\left(z_{0}\right)\right|}{\left|2 A-f\left(z_{0}\right)\right|^{2}} .
$$

Since $\left|2 A-f\left(z_{0}\right)\right|^{2} \geq\left(\Re\left(2 A-f\left(z_{0}\right)\right)\right)^{2}=A^{2}$, we get

$$
\frac{2\left(4 A^{2}-\left|c_{p}\right|^{2}\right)}{4 A^{2}-\left|c_{p}\right|^{2}+2 A\left|c_{p+1}\right|} \frac{2 A-\left|c_{p}\right|}{2 A+\left|c_{p}\right|}+p \leq \frac{2 A\left|f^{\prime}\left(z_{0}\right)\right|}{\left|2 A-f\left(z_{0}\right)\right|^{2}} \leq \frac{2\left|f^{\prime}\left(z_{0}\right)\right|}{A}
$$

and

$$
\left(\frac{2\left(2 A-\left|c_{p}\right|\right)^{2}}{4 A^{2}-\left|c_{p}\right|^{2}+2 A\left|c_{p+1}\right|}+p\right) \frac{A}{2} \leq\left|f^{\prime}\left(z_{0}\right)\right| .
$$

So, we get inequality (1.6) .

Now we shall show that inequality (1.6) is sharp. Let

$$
f(z)=2 A \frac{z^{p}}{1+z^{p}} .
$$

Then

$$
f^{\prime}(z)=2 A \frac{p z^{p-1}}{\left(1+z^{p}\right)^{2}}
$$

and

$$
f^{\prime}(1)=\frac{p A}{2}
$$

Since $\left|c_{p}\right|=2 A,(1.6)$ holds.

Theorem 2. Let $f(z)=c_{p} z^{p}+c_{p+1} z^{p+1} \ldots, c_{p}>0, p \geq 2, p \in, \mathbb{N}$, be a holomorphic function in the unit disc $D$ and $f(z)$ have no zeros in $D$ except $z=0$, and let $\Re f(z) \leqslant A$ for $|z|<1$. Further, assume that for some $z_{0} \in \partial D$, 
$f$ has an angular limit $f\left(z_{0}\right)$ at $z_{0}, \Re f\left(z_{0}\right)=A$. Then the angular derivative $f^{\prime}\left(z_{0}\right)$ exists and

$$
\left|f^{\prime}\left(z_{0}\right)\right| \geqslant \frac{A}{2}\left(p-\frac{2\left|c_{p}\right|\left(\ln \frac{\left|c_{p}\right|}{2 A}\right)^{2}}{2\left|c_{p}\right| \ln \left(\frac{\left|c_{p}\right|}{2 A}\right)-\left|c_{p+1}\right|}\right),
$$

where

$$
\left|c_{p+1}\right| \leq 2\left|c_{p} \ln \left(\frac{\left|c_{p}\right|}{2 A}\right)\right|
$$

In addition, the equality in (1.9) occurs for the function $f(z)=2 A \frac{z^{p}}{1+z^{p}}$, and the equality in (1.10) occurs for the function

$$
f(z)=2 A \frac{z^{p} e^{\frac{1+z}{1-z} \ln \left(\frac{c_{p}}{2 A}\right)}}{1+z^{p} e^{\frac{1+z}{1-z} \ln \left(\frac{c_{p}}{2 A}\right)}},
$$

where $0<c_{p}<1$ and $\ln \left(\frac{c_{p}}{2 A}\right)<0$.

P r o o f. Let $c_{p}>0$. Let $w(z), t(z)$ and $B(z)$ be as in the proof of Theorem 1. Having in mind inequality (1.7), we denote by $\ln t(z)$ the holomorphic branch of the logarithm normed by the condition

$$
\ln t(0)=\ln \left(\frac{c_{p}}{2 A}\right)<0
$$

The function

$$
b(z)=\frac{\ln t(z)-\ln t(0)}{\ln t(z)+\ln t(0)}
$$

is holomorphic in the unit disc $D,|b(z)|<1, b(0)=0$, and $\left|b\left(z_{0}\right)\right|=1$ for $z_{0} \in \partial D$. Since $f(z)$ has an angular limit at $z_{0}, w(z)$ has an angular limit at $z_{0}$ and from Julia-Wolff lemma the function $w(z)$ has an angular derivative at $z_{0}$. Thus, since $w(z)$ has an angular derivative at $z_{0}$, the function $f(z)$ has an angular derivative at $z_{0}$. From (1.3), we obtain

$$
\begin{aligned}
\frac{2}{1+\left|b^{\prime}(0)\right|} & \leq\left|b^{\prime}\left(z_{0}\right)\right|=\frac{|2 \ln t(0)|}{\left|\ln t\left(z_{0}\right)+\ln t(0)\right|^{2}}\left|\frac{t^{\prime}\left(z_{0}\right)}{t\left(z_{0}\right)}\right| \\
& =\frac{|2 \ln t(0)|}{\left|\ln t\left(z_{0}\right)+\ln t(0)\right|^{2}}\left|t^{\prime}\left(z_{0}\right)\right| \\
& =\frac{|2 \ln t(0)|}{\left|\ln t\left(z_{0}\right)+\ln t(0)\right|^{2}}\left|\frac{w^{\prime}\left(z_{0}\right)}{B\left(z_{0}\right)}-\frac{w\left(z_{0}\right) B^{\prime}\left(z_{0}\right)}{B^{2}\left(z_{0}\right)}\right| \\
& =\frac{-2 \ln t(0)}{\ln ^{2} t(0)+\arg ^{2} t\left(z_{0}\right)}\left\{\left|w^{\prime}\left(z_{0}\right)\right|-\left|B^{\prime}\left(z_{0}\right)\right|\right\}
\end{aligned}
$$


and

$$
\frac{2}{1+\left|b^{\prime}(0)\right|} \leq \frac{-2 \ln t(0)}{\ln ^{2} t(0)+\arg ^{2} t\left(z_{0}\right)}\left\{\left|w^{\prime}\left(z_{0}\right)\right|-\left|B^{\prime}\left(z_{0}\right)\right|\right\} .
$$

It can be seen that

$$
b^{\prime}(z)=\frac{2 \ln t(0)}{(\ln t(z)+\ln t(0))^{2}} \frac{t^{\prime}(z)}{t(z)}
$$

and

$$
\left|b^{\prime}(0)\right|=\frac{1}{|2 \ln t(0)|}\left|\frac{t^{\prime}(0)}{t(0)}\right|=\frac{1}{-2 \ln \frac{\left|c_{p}\right|}{2 A}} \frac{\left|c_{p+1}\right|}{\left|c_{p}\right|} .
$$

Let us substitute the values of $\left|b^{\prime}(0)\right|,\left|w^{\prime}\left(z_{0}\right)\right|,\left|B^{\prime}\left(z_{0}\right)\right|$ and $\ln t(0)$ into (1.11). We obtain

$$
\frac{2}{1-\frac{\left|c_{p+1}\right|}{2\left|c_{p}\right| \ln \left(\frac{\left|c_{p}\right|}{2 A}\right)}} \leq \frac{-2 \ln t(0)}{\ln ^{2} t(0)+\arg ^{2} t\left(z_{0}\right)}\left\{\frac{2 A\left|f^{\prime}\left(z_{0}\right)\right|}{\left|2 A-f\left(z_{0}\right)\right|^{2}}-p\right\} .
$$

Replacing $\arg ^{2} t\left(z_{0}\right)$ by zero, we have

$$
\frac{2}{1-\frac{\left|c_{p+1}\right|}{2\left|c_{p}\right| \ln \left(\frac{\left|c_{p}\right|}{2 A}\right)}} \leq \frac{-2}{\ln t(0)}\left\{\frac{2 A\left|f^{\prime}\left(z_{0}\right)\right|}{\left|2 A-f\left(z_{0}\right)\right|^{2}}-p\right\}
$$

and

$$
\frac{2\left|c_{p}\right| \ln \left(\frac{\left|c_{p}\right|}{2 A}\right)}{2\left|c_{p}\right| \ln \left(\frac{\left|c_{p}\right|}{2 A}\right)-\left|c_{p+1}\right|} \leq \frac{-1}{\ln \left(\frac{\left|c_{p}\right|}{2 A}\right)}\left\{\frac{2 A\left|f^{\prime}\left(z_{0}\right)\right|}{\left|2 A-f\left(z_{0}\right)\right|^{2}}-p\right\} .
$$

Since $\left|2 A-f\left(z_{0}\right)\right|^{2} \geq\left(\Re\left(2 A-f\left(z_{0}\right)\right)\right)^{2}=A^{2}$, we get

$$
p-\frac{2\left|c_{p}\right|\left(\ln \frac{\left|c_{p}\right|}{2 A}\right)^{2}}{2\left|c_{p}\right| \ln \left(\frac{\left|c_{p}\right|}{2 A}\right)-\left|c_{p+1}\right|} \leq \frac{2 A\left|f^{\prime}\left(z_{0}\right)\right|}{\left|2 A-f\left(z_{0}\right)\right|^{2}} \leq \frac{2\left|f^{\prime}\left(z_{0}\right)\right|}{A} .
$$

Thus, we obtain (1.9) with an obvious equality case.

Similarly, the function $b(z)$ satisfies the assumptions of the Schwarz lemma ([5]), we obtain

$$
1 \geq\left|b^{\prime}(0)\right|=\frac{|2 \ln t(0)|}{|\ln t(0)+\ln t(0)|^{2}}\left|\frac{t^{\prime}(0)}{t(0)}\right|
$$

and

$$
1 \geq \frac{-1}{2 \ln \left(\frac{\left|c_{p}\right|}{2 A}\right)} \frac{\left|c_{p+1}\right|}{\left|c_{p}\right|}
$$


Therefore, we have inequality (1.10).

Now we shall show that inequality (1.10) is sharp. Let

$$
f(z)=z^{p} g(z),
$$

where

$$
g(z)=2 A \frac{e^{\frac{1+z}{1-z} \ln \left(\frac{c_{p}}{2 A}\right)}}{1+z^{p} e^{\frac{1+z}{1-z} \ln \left(\frac{c_{p}}{2 A}\right)}} .
$$

Then

$$
g(0)=c_{p}
$$

and

$$
g^{\prime}(0)=c_{p+1} .
$$

After simple calculations, we get

$$
c_{p+1}=2 c_{p} \ln \left(\frac{c_{p}}{2 A}\right) .
$$

Thus we obtain

$$
\left|c_{p+1}\right|=2\left|c_{p} \ln \left(\frac{\left|c_{p}\right|}{2 A}\right)\right|
$$

We note that inequality (1.3) has been used in the proofs of Theorems 1 and 2. Therefore, there are both $c_{p}$ and $c_{p+1}$ in the right-hand side of the inequalities. But, if we use (1.4) instead of (1.3), we obtain a weaker but simpler inequality (not including $c_{p+1}$ ). It is formulated in the following theorem.

Theorem 3. Under the hypotheses of Theorem 2, we have

$$
\left|f^{\prime}\left(z_{0}\right)\right| \geqslant \frac{A}{2}\left(p-\frac{1}{2} \ln \frac{\left|c_{p}\right|}{2 A}\right) .
$$

The equality in (1.12) holds if and only if

$$
f(z)=2 A \frac{z^{p} e^{\frac{1+z e^{i \theta}}{1-z e^{i \theta}} \ln \left(\frac{c_{p}}{2 A}\right)}}{1+z^{p} e^{\frac{1+z e e^{i \theta}}{1-z e^{i \theta}} \ln \left(\frac{c_{p}}{2 A}\right)},},
$$

where $0<c_{p}<1, \ln \left(\frac{c_{p}}{2 A}\right)<0$, and $\theta$ is a real number. 
P r o o f. From the proof of Theorem 2, using inequality (1.4) for the function $b(z)$, we obtain

$$
\begin{aligned}
1 & \leq\left|b^{\prime}\left(z_{0}\right)\right|=\frac{|2 \ln t(0)|}{\left|\ln t\left(z_{0}\right)+\ln t(0)\right|^{2}}\left|\frac{t^{\prime}\left(z_{0}\right)}{t\left(z_{0}\right)}\right| \\
& =\frac{-2 \ln t(0)}{\ln ^{2} t(0)+\arg ^{2} t\left(z_{0}\right)}\left\{\frac{2 A\left|f^{\prime}\left(z_{0}\right)\right|}{\left|2 A-f\left(z_{0}\right)\right|^{2}}-p\right\} .
\end{aligned}
$$

Replacing $\arg ^{2} t\left(z_{0}\right)$ by zero and since $\left|2 A-f\left(z_{0}\right)\right|^{2} \geq\left(\Re\left(2 A-f\left(z_{0}\right)\right)\right)^{2}=A^{2}$, we get

$$
1 \leq\left|b^{\prime}\left(z_{0}\right)\right| \leq \frac{-2}{\ln \left(\frac{\left|c_{p}\right|}{2 A}\right)}\left(\frac{2}{A}\left|f^{\prime}\left(z_{0}\right)\right|-p\right) .
$$

Therefore, we have inequality (1.12).

$$
\begin{gathered}
\text { If }\left|f^{\prime}\left(z_{0}\right)\right|=\frac{A}{2}\left(p-\frac{1}{2} \ln \frac{\left|c_{p}\right|}{2 A}\right) \text {, from }(1.13) \text { and }\left|b^{\prime}\left(z_{0}\right)\right|=1 \text { we obtain } \\
b(z)=z e^{i \theta}
\end{gathered}
$$

and

$$
\frac{\ln t(z)-\ln t(0)}{\ln t(z)+\ln t(0)}=z e^{i \theta}
$$

Therefore, we take

$$
\begin{aligned}
\ln t(z) & =\frac{1+z e^{i \theta}}{1-z e^{i \theta}} \ln \left(\frac{c_{p}}{2 A}\right), \\
t(z) & =e^{\frac{1+z e^{i \theta}}{1-z e^{i \theta}} \ln \left(\frac{c_{p}}{2 A}\right)}, \\
\frac{w(z)}{B(z)} & =e^{\frac{1+z e^{i \theta}}{1-z e^{i \theta}} \ln \left(\frac{c_{p}}{2 A}\right)}, \\
\frac{f(z)}{2 A-f(z)} & =z^{p} e^{\frac{1+z e^{i \theta}}{1-z e^{i \theta}} \ln \left(\frac{c_{p}}{2 A}\right)}
\end{aligned}
$$

and

$$
f(z)=2 A \frac{z^{p} e^{\frac{1+z e^{i \theta}}{1-z e^{i \theta}} \ln \left(\frac{c_{p}}{2 A}\right)}}{1+z^{p} e^{\frac{1+z e i \theta}{1-z e^{i \theta}} \ln \left(\frac{c_{p}}{2 A}\right)}} .
$$

Consider the product

$$
B_{n}(z)=\prod_{k=1}^{n} \frac{z-a_{k}}{1-\overline{a_{k}} z}
$$


The function $B_{n}(z)$ is called a finite Blaschke product, where $a_{1}, a_{2}, \ldots, a_{n} \in$ $D$. Let the function $f(z)$ satisfy the conditions of the Carathéodory inequality and also have zeros $a_{1}, a_{2}, \ldots, a_{n}$ with order $n_{1}, n_{2}, \ldots . n_{k}$, respectively. Thus, one can see that the Carathéodory inequality can be strengthened by standard methods as follows:

$$
|f(z)| \leq \frac{2 A|z|^{p}\left|B_{n}(z)\right|}{1-|z|^{p}\left|B_{n}(z)\right|}
$$

and

$$
\left|c_{p}\right| \leq 2 A \prod_{k=1}^{n}\left|a_{k}\right| .
$$

Inequalities (1.14) and (1.15) show that inequalities (1.1) and (1.2) can be strengthened if the zeros of the function which are different from the origin of $f(z)$ in inequality (1.6) are taken into account.

Theorem 4. Let $f(z)=c_{p} z^{p}+c_{p+1} z^{p+1} \ldots, c_{p} \neq 0, p \geq 2, p \in \mathbb{N}$, be a holomorphic function in the unit disc $D$ and let $\Re f(z) \leqslant A$ for $|z|<1$. Assume that for some $z_{0} \in \partial D, f$ has an angular limit $f\left(z_{0}\right)$ at $z_{0}, \Re f\left(z_{0}\right)=A$. Let $a_{1}, a_{2}, \ldots, a_{n}$ be the zeros of the function $f(z)$ in $D$ that are different from zero. Then the angular derivative $f^{\prime}\left(z_{0}\right)$ exists, and

$$
\left|f^{\prime}\left(z_{0}\right)\right| \geqslant \frac{A}{2}\left\{p+\sum_{k=1}^{n} \frac{1-\left|a_{k}\right|^{2}}{\left|z_{0}-a_{k}\right|^{2}}+\frac{2\left(2 A \prod_{k=1}^{n}\left|a_{k}\right|-\left|c_{p}\right|\right)^{2}}{4 A^{2}\left(\prod_{k=1}^{n}\left|a_{k}\right|\right)^{2}-\left|c_{p}\right|^{2}+2 A\left|c_{p+1}\right| \prod_{k=1}^{n}\left|a_{k}\right|}\right\} .
$$

In addition, the equality in (1.16) occurs for the function

$$
f(z)=2 A\left(1-\frac{1}{1+z^{p} \prod_{k=1}^{n} \frac{z-a_{k}}{1-\overline{a_{k}} z}}\right),
$$

where $a_{1}, a_{2}, \ldots, a_{n}$ are positive real numbers.

P r o o f. Let $w(z)$ be as in the proof of Theorem 1 and $a_{1}, a_{2}, \ldots, a_{n}$ be the zeros of the function $f(z)$ in $D$ that are different from zero. The function

$$
B_{1}(z)=z^{p} \prod_{k=1}^{n} \frac{z-a_{k}}{1-\overline{a_{k}} z}
$$


is holomorphic in $D$, and $\left|B_{1}(z)\right|<1$ for $|z|<1$. By the maximum principle, for each $z \in D$, we have

$$
|w(z)| \leq\left|B_{1}(z)\right|
$$

The function

$$
t_{1}(z)=\frac{w(z)}{B_{1}(z)}
$$

is holomorphic in $D$, and $\left|t_{1}(z)\right| \leq 1$ for $|z| \leq 1$. In particular, we have

$$
\left|t_{1}(0)\right|=\frac{\left|c_{p}\right|}{2 A \prod_{k=1}^{n}\left|a_{k}\right|} \leq 1
$$

and

$$
\left|t_{1}^{\prime}(0)\right|=\frac{\left|c_{p+1}\right|}{2 A \prod_{k=1}^{n}\left|a_{k}\right|} .
$$

If $\left|t_{1}(0)\right|=1$, then, by the maximum principle, we have $\frac{w(z)}{B_{1}(z)}=e^{i \varphi}$ and

$$
f(z)=2 A \frac{z^{p} e^{i \varphi} \prod_{k=1}^{n} \frac{z-a_{k}}{1-\overline{a_{k}} z}}{1+z^{p} e^{i \varphi} \prod_{k=1}^{n} \frac{z-a_{k}}{1-\overline{a_{k}} z}},
$$

where $\varphi$ is a real number. For the function $f(z),(1.16)$ holds. Thus we may assume

$$
f(z) \not \equiv 2 A \frac{z^{p} e^{i \varphi} \prod_{k=1}^{n} \frac{z-a_{k}}{1-\overline{a_{k}} z}}{1+z^{p} e^{i \varphi} \prod_{k=1}^{n} \frac{z-a_{k}}{1-\overline{a_{k}} z}},
$$

and $\left|t_{1}(0)\right|<1$.

It is obvious that

$$
\left|B_{1}^{\prime}\left(z_{0}\right)\right|=\frac{z_{0} B_{1}^{\prime}\left(z_{0}\right)}{B_{1}\left(z_{0}\right)}=p+\sum_{k=1}^{n} \frac{1-\left|a_{k}\right|^{2}}{\left|z_{0}-a_{k}\right|^{2}} .
$$

Furthermore, since the expression $\frac{z_{0} w^{\prime}\left(z_{0}\right)}{w\left(z_{0}\right)}$ is a real number greater than or equal to 1 (see [7]) and $\Re f\left(z_{0}\right)=A$ yields $\left|w\left(z_{0}\right)\right|=1$, we get

$$
\frac{z_{0} w^{\prime}\left(z_{0}\right)}{w\left(z_{0}\right)}=\left|\frac{z_{0} w^{\prime}\left(z_{0}\right)}{w\left(z_{0}\right)}\right|=\left|w^{\prime}\left(z_{0}\right)\right| .
$$


Also, since $|w(z)| \leq\left|B_{1}(z)\right|$, we take

$$
\frac{1-|w(z)|}{1-|z|} \geq \frac{1-\left|B_{1}(z)\right|}{1-|z|}
$$

Since $f(z)$ has an angular limit at $z_{0}, w(z)$ has an angular limit at $z_{0}$ and from Julia-Wolff lemma the function $w(z)$ has an angular derivative at $z_{0}$. Passing to the angular limit in the last inequality yields

$$
\left|w^{\prime}\left(z_{0}\right)\right| \geq\left|B_{1}^{\prime}\left(z_{0}\right)\right|
$$

Therefore, we obtain

$$
\frac{z_{0} w^{\prime}\left(z_{0}\right)}{w\left(z_{0}\right)}=\left|w^{\prime}\left(z_{0}\right)\right| \geq\left|B_{1}^{\prime}\left(z_{0}\right)\right|=\frac{z_{0} B_{1}^{\prime}\left(z_{0}\right)}{B_{1}\left(z_{0}\right)} .
$$

The auxiliary function

$$
T_{1}(z)=\frac{t_{1}(z)-t_{1}(0)}{1-\overline{t_{1}(0)} t_{1}(z)}
$$

is holomorphic in the unit disc $D,\left|T_{1}(z)\right|<1, T_{1}(0)=0$, and $\left|T_{1}\left(z_{0}\right)\right|=1$ for $z_{0} \in \partial D$. From (1.3), we obtain

$$
\begin{aligned}
\frac{2}{1+\left|T_{1}^{\prime}(0)\right|} & \leq\left|T_{1}^{\prime}\left(z_{0}\right)\right|=\frac{1-\left|t_{1}(0)\right|^{2}}{\left|1-\overline{t_{1}(0)} t_{1}\left(z_{0}\right)\right|^{2}}\left|t_{1}^{\prime}\left(z_{0}\right)\right| \\
& =\frac{1-\left|t_{1}(0)\right|^{2}}{\left|1-\overline{t_{1}(0)} t_{1}\left(z_{0}\right)\right|^{2}}\left|\frac{w^{\prime}\left(z_{0}\right)}{B_{1}\left(z_{0}\right)}-\frac{w\left(z_{0}\right) B_{1}^{\prime}\left(z_{0}\right)}{B_{1}^{2}\left(z_{0}\right)}\right| \\
& \leq \frac{1+\left|t_{1}(0)\right|}{1-\left|t_{1}(0)\right|}\left\{\left|w^{\prime}\left(z_{0}\right)\right|-\left|B_{1}^{\prime}\left(z_{0}\right)\right|\right\}
\end{aligned}
$$

and

$$
\frac{2}{1+\left|T_{1}^{\prime}(0)\right|} \leq \frac{1+\left|t_{1}(0)\right|}{1-\left|t_{1}(0)\right|}\left\{\left|w^{\prime}\left(z_{0}\right)\right|-\left|B_{1}^{\prime}\left(z_{0}\right)\right|\right\} .
$$

It can be seen that

$$
T_{1}^{\prime}(z)=\frac{1-\left|t_{1}(0)\right|^{2}}{\left(1-\overline{t_{1}(0)} t_{1}(z)\right)^{2}} t_{1}^{\prime}(z)
$$

and

$$
\left|T_{1}^{\prime}(0)\right|=\frac{\left|t_{1}^{\prime}(0)\right|}{1-\left|t_{1}(0)\right|^{2}}=\frac{\frac{\left|c_{p+1}\right|}{2 A \prod_{k=1}^{n}\left|a_{k}\right|}}{1-\left(\frac{\left|c_{p}\right|}{2 A \prod_{k=1}^{n}\left|a_{k}\right|}\right)^{2}}=\frac{2 A\left|c_{p+1}\right| \prod_{k=1}^{n}\left|a_{k}\right|}{4 A^{2}\left(\prod_{k=1}^{n}\left|a_{k}\right|\right)^{2}-\left|c_{p}\right|^{2}} .
$$


Since $w(z)$ has an angular derivative at $z_{0}$, the function $f(z)$ has an angular derivative at $z_{0}$. Let us substitute the values of $\left|T_{1}^{\prime}(0)\right|,\left|w^{\prime}\left(z_{0}\right)\right|,\left|B_{1}^{\prime}\left(z_{0}\right)\right|$ and $\left|t_{1}(0)\right|$ into (1.17). Thus, we obtain

$$
\frac{2}{1+\frac{2 A\left|c_{p+1}\right| \prod_{k=1}^{n}\left|a_{k}\right|}{4 A^{2}\left(\prod_{k=1}^{n}\left|a_{k}\right|\right)^{2}-\left|c_{p}\right|^{2}}} \leq \frac{2 A \prod_{k=1}^{n}\left|a_{k}\right|+\left|c_{p}\right|}{2 A \prod_{k=1}^{n}\left|a_{k}\right|-\left|c_{p}\right|}\left\{\frac{2 A\left|f^{\prime}\left(z_{0}\right)\right|}{\left|2 A-f\left(z_{0}\right)\right|^{2}}-p-\sum_{k=1}^{n} \frac{1-\left|a_{k}\right|^{2}}{\left|z_{0}-a_{k}\right|^{2}}\right\}
$$

$$
\frac{2\left[4 A^{2}\left(\prod_{k=1}^{n}\left|a_{k}\right|\right)^{2}-\left|c_{p}\right|^{2}\right]}{4 A^{2}\left(\prod_{k=1}^{n}\left|a_{k}\right|\right)^{2}-\left|c_{p}\right|^{2}+2 A\left|c_{p+1}\right| \prod_{k=1}^{n}\left|a_{k}\right|} \frac{2 A \prod_{k=1}^{n}\left|a_{k}\right|-\left|c_{p}\right|}{2 A \prod_{k=1}^{n}\left|a_{k}\right|+\left|c_{p}\right|}+p+\sum_{k=1}^{n} \frac{1-\left|a_{k}\right|^{2}}{\left|z_{0}-a_{k}\right|^{2}} \leq \frac{2 A\left|f^{\prime}\left(z_{0}\right)\right|}{\left|2 A-f\left(z_{0}\right)\right|^{2}}
$$

and since $\left|2 A-f\left(z_{0}\right)\right|^{2} \geq\left(\Re\left(2 A-f\left(z_{0}\right)\right)\right)^{2}=A^{2}$, we get

$$
\frac{2\left(2 A \prod_{k=1}^{n}\left|a_{k}\right|-\left|c_{p}\right|\right)^{2}}{4 A^{2}\left(\prod_{k=1}^{n}\left|a_{k}\right|\right)^{2}-\left|c_{p}\right|^{2}+2 A\left|c_{p+1}\right| \prod_{k=1}^{n}\left|a_{k}\right|}+p+\sum_{k=1}^{n} \frac{1-\left|a_{k}\right|^{2}}{\left|z_{0}-a_{k}\right|^{2}} \leq \frac{2\left|f^{\prime}\left(z_{0}\right)\right|}{A} .
$$

Hence we get inequality (1.16).

Now we shall show that inequality (1.16) is sharp. Let

$$
f(z)=2 A\left(1-\frac{1}{1+z^{p} \prod_{k=1}^{n} \frac{z-a_{k}}{1-\bar{a}_{k} z}}\right) .
$$

Then

$$
f^{\prime}(z)=2 A\left(\frac{p z^{p-1} \prod_{k=1}^{n} \frac{z-a_{k}}{1-\overline{a_{k}} z}+\sum_{k=1}^{n} \frac{1-\left|a_{k}\right|^{2}}{\left(1-\overline{a_{k}} z\right)^{2}} \prod_{\substack{s=1 \\ k \neq s}}^{n} \frac{z-a_{s}}{1-\overline{a_{s}} z} z^{p}}{\left(1+z^{p} \prod_{k=1}^{n} \frac{z-a_{k}}{1-\overline{a_{k}} z}\right)^{2}}\right)
$$

and

$$
f^{\prime}(1)=2 A\left(\frac{p \prod_{k=1}^{n} \frac{1-a_{k}}{1-\overline{a_{k}}}+\sum_{k=1}^{n} \frac{1-\left|a_{k}\right|^{2}}{\left(1-\overline{a_{k}}\right)^{2}} \prod_{\substack{s=1 \\ k \neq s}}^{n} \frac{1-a_{s}}{1-\overline{a_{s}}}}{\left(1+\prod_{k=1}^{n} \frac{1-a_{k}}{1-\overline{a_{k}}}\right)^{2}}\right) \text {. }
$$


Since $a_{1}, a_{2}, \ldots, a_{n}$ are positive real numbers, we have

$$
f^{\prime}(1)=\frac{2 A}{4}\left(p+\sum_{k=1}^{n} \frac{1-a_{k}^{2}}{\left(1-a_{k}\right)^{2}}\right)=\frac{A}{2}\left(p+\sum_{k=1}^{n} \frac{1+a_{k}}{1-a_{k}}\right) .
$$

Moreover, since $\left|c_{p}\right|=2 A \prod_{k=1}^{n}\left|a_{k}\right|,(1.16)$ holds.

Acknowledgement. The author would like to thank the referees for their constructive comments and suggestions on the earlier version of this paper.

\section{References}

[1] H.P. Boas, Julius and Julia: Mastering the Art of the Schwarz Lemma. - American Mathematical Monthly 117 (2010), No. 9, 770-785.

[2] D.M. Burns and S.G. Krantz, Rigidity of Holomorphic Mappings and a New Schwarz Lemma at the Boundary. - J. Amer. Math. Soc. 7 (1994), 661-676.

[3] C. Carathéodory, Theory of Functions. Vol. 2. Chelsea, New York, 1954.

[4] V.N. Dubinin, The Schwarz Inequality on the Boundary for Functions Regular in the Disc. - J. Math. Sci. 122 (2004), No. 2, 3623-3629.

[5] G.M. Golusin, Geometric Theory of Functions of Complex Variable. 2nd edn. Moscow, 1966. (Russian)

[6] G. Kresin and V. Maz'ya, Sharp Real-Part Theorems. A Unified Approach., Translated from Russian and edited by T. Shaposhnikova. Lecture Notes in Mathematics, 1903. Springer, Berlin, 2007.

[7] R. Osserman, A Sharp Schwarz Inequality on the Boundary. - Proc. Amer. Math. Soc. 128 (2000), 3513-3517.

[8] B.N. Örnek, A Sharp Schwarz and Carathéodory Inequality on the Boundary. Commun. Korean Math. Soc. 29 (2014), No. 1, 75-81.

[9] Ch. Pommerenke, Boundary Behaviour of Conformal Maps. Springer-Verlag, Berlin, 1992. 